LAWRENCE LIVERMORE N A T IO N A L LABORATORY

\title{
A Generalized Mass Lumping Scheme for Maxwell's Wave Equation
}

A. Fisher, D. White, G. Rodrigue

January 20, 2004

2004 IEEE AP-S International Symposium and USNC / URSI National Radio Science Meeting Monterey, CA, United States June 20, 2004 through June 26, 2004 
This document was prepared as an account of work sponsored by an agency of the United States Government. Neither the United States Government nor the University of California nor any of their employees, makes any warranty, express or implied, or assumes any legal liability or responsibility for the accuracy, completeness, or usefulness of any information, apparatus, product, or process disclosed, or represents that its use would not infringe privately owned rights. Reference herein to any specific commercial product, process, or service by trade name, trademark, manufacturer, or otherwise, does not necessarily constitute or imply its endorsement, recommendation, or favoring by the United States Government or the University of California. The views and opinions of authors expressed herein do not necessarily state or reflect those of the United States Government or the University of California, and shall not be used for advertising or product endorsement purposes. 


\title{
A Generalized Mass Lumping Scheme for Maxwell's Wave Equation
}

\author{
Aaron Fisher*1, Daniel White ${ }^{2}$, Garry Rodrigue ${ }^{1}$ \\ 1: Department of Applied Science, University of California Davis \\ 2: Lawrence Livermore National Laboratory"
}

\section{Introduction}

We are interested in the high order Vector Finite Element Method (VFEM) [1] solution to Maxwell's wave equation on both orthogonal and non-orthogonal meshes. This method discretizes the wave equation in the following manner, where $\mathrm{M}$ is the edge mass matrix and $\mathrm{K}$ is the edge stiffness matrix created using classical Nedelec edge elements [2].

$$
\begin{gathered}
\frac{d^{2} \varepsilon \widehat{E}}{d t^{2}}=-\nabla \times \mu^{-1} \nabla \times \widehat{E} \\
M \frac{d^{2}}{d t^{2}} e=-K e
\end{gathered}
$$

This method has the advantage of being able to model complicated geometries while retaining a high degree of accuracy when compared to traditional FE and FDTD approaches. On the other hand this method is very computationally expensive due to the need to invert very large and relatively dense mass matrices. However, we present a generalized mass lumping scheme which allows us to greatly reduce the computational expense of the mass matrix 'inversion' without a loss of accuracy. In the orthogonal case the mass matrix inversion is all but eliminated, and in the non-orthogonal case the computational cost is reduced by more than a factor of 10 . This method can also take full advantage of meshes which have some orthogonal elements and some non-orthogonal elements.

There are currently existing methods that provide high order solutions while mitigating the computational cost of 'inverting' the mass matrix. Most notable are the Finite Volume methods and the Gauss Point Mass Lumping Schemes [3]. These methods reduce the computational by creating block diagonal mass matrices. However, neither of these methods use the classical Nedelec edge elements to model the electric field.

Consequently, these methods introduce spurious modes into their solutions making them unsuitable for problems where eigenvalues are important.

\section{Directional Mass Lumping}

Normal mass lumping methods use a carefully constructed integration rule for all of the mass integrals in order to create a diagonal mass matrix. There are complications that arise when applying this approach to the VFEM, so a generalized approach is applied. Instead of relying on a single integration rule to compute the mass integrals involving all of the basis function combinations, multiple integration rules are constructed to compute mass integrals for different combinations of basis functions. With this added freedom, integration rules can be constructed to increase sparsity for particular sets of basis interactions.

In general the Nedelec bases can be made from Lagrange interpolatory polynomials. The precise definition of each of these polynomials is given by:

$$
L_{i}^{p}(x, Q)=\prod_{j=0, j \neq i}^{p} \frac{\left(x-q_{j}\right)}{\left(q_{i}-q_{j}\right.}
$$

\# UCRL-CONF-201900 
where $\mathrm{p}$ is the degree of each of the $\mathrm{i}^{\text {th }}$ polynomials, and $\mathrm{Q}=\left\{\mathrm{q}_{0}, \mathrm{q}_{1}, \mathrm{q}_{2}, \ldots, \mathrm{q}_{\mathrm{p}}\right\}$ is a set of interpolation points. Using these polynomials, the Nedelec hexahedral bases can be constructed in three different sets $(\mathrm{a}, \mathrm{b}, \mathrm{c})$ by the following equations.

$$
\begin{gathered}
\widehat{\widehat{\phi_{i j k}^{a}}}(x, y, z)=L_{i}^{p-1}\left(x, Q_{1}\right) L_{j}^{p}\left(y, Q_{2}\right) L_{k}^{p}\left(z, Q_{3}\right) \widehat{\boldsymbol{x}} \\
\widehat{\phi_{i j k}^{b}}(x, y, z)=L_{j}^{p}\left(x, Q_{2}\right) L_{i}^{p-1}\left(y, Q_{1}\right) L_{k}^{p}\left(z, Q_{3}\right) \widehat{\boldsymbol{y}} \\
\widehat{\phi_{i j k}^{c}}(x, y, z)=L_{j}^{p}\left(x, Q_{2}\right) L_{k}^{p}\left(y, Q_{3}\right) L_{i}^{p-1}\left(z, Q_{1}\right) \widehat{z} \\
\text { For all romhinations of } i=01 \quad n-1 \quad i \quad k=01 \quad n
\end{gathered}
$$

This description leaves many options open for what sets of interpolation points may be used. However, for reasons that will become apparent, we choose the Gauss-Legendre $(G)$ quadrature points on $[0,1]$ for $\mathrm{Q}_{1}$ and the Gauss-Lobatto $(\mathrm{B})$ quadrature points on $[0,1]$ for $\mathrm{Q}_{2}$ and $\mathrm{Q}_{3}$.

With the description of the basis functions complete the mass integrals can be formed in four different categories. The (a), (b) and (c) integrals only work with pairs of basis functions that point in the $\mathrm{x}, \mathrm{y}$, and $\mathrm{z}$ directions respectively. The $\mathrm{d}$ integrals work with pairs of basis functions that point in differing directions. The mass integrals in each category can be used to create four mass matrices that have entries relevant to their respective categories and are zero elsewhere. Finally, these four matrices are added together to create the element mass matrix as follows.

$$
\begin{aligned}
M_{l m}^{a}= & \int_{V} \varepsilon \widehat{\phi_{l}^{a}} \cdot \widehat{\phi_{m}^{a}} d V \quad M_{l m}^{b}=\int_{V} \varepsilon \widehat{\phi_{l}^{b}} \cdot \widehat{\phi_{m}^{b}} d V \\
M_{l m}^{c}= & \int_{V} \varepsilon \widehat{\phi_{l}^{c}} \cdot \widehat{\phi_{m}^{c}} d V \quad M_{l m}^{d}=\int_{V} \varepsilon \widehat{\phi_{l}^{d}} \cdot \widehat{\phi_{m}^{d^{\prime}}} d V \\
& \text { where } m, n \in\{a, b, c\} \text { with } d \neq d^{\prime}
\end{aligned}
$$

Each of these four categories of integrals will be assigned an integration rule, however it is instructive at this point to consider a property of the Lagrange interpolatory polynomials. It can be easily shown that the following equation holds.

$$
L_{i}\left(q_{i}, Q\right)=0 \quad \text { for } i \neq j
$$

Given this equation, natural choices of integration points for the (a), (b), and (c) mass integrals arise. If any of the integration points coincide with the interpolation points of the basis functions then by the above result increased sparsity can be expected. In particular, for the (a), (b), and (c) mass integrals the integration rule can be defined on the set of points described by the tensor product of the interpolation points in each dimension. Since we earlier chose the interpolation points to be Gauss-Legendre and Gauss-Lobatto on $[0,1]$ the integration rules are simply Gauss-Legendre integration in one of the dimensions and Gauss-Lobatto integration in the other two dimensions. This set of integration points takes full advantage of the above result in the following manner.

$$
\begin{gathered}
\xi_{r s t}^{a}=\left(g_{r}, b_{s}, b_{t}\right) \quad \text { where } g_{r} \in G \quad b_{s}, b_{t} \in B \\
\widehat{\phi_{i j k}^{a}}\left(\xi_{r s t}^{a}\right)=L_{i}^{p-1}\left(g_{r}, G\right) L_{j}^{p}\left(b_{s}, B\right) L_{k}^{p}\left(b_{t}, Q\right) \widehat{\boldsymbol{x}}=0 \quad \text { for } i j k \neq 1 \\
M_{l m}^{a}=\int_{V} \varepsilon \widehat{\phi_{l}^{a}} \cdot \widehat{\phi_{m}^{a}} d V \simeq \sum_{r s t} w_{r s t} \varepsilon \widehat{\phi_{i j k}^{a}}\left(\xi_{r s t}^{a}\right) \cdot \widehat{\phi_{i^{\prime} j^{\prime} k^{\prime}}^{a}}\left(\xi_{r s t}^{a}\right)=0 \\
\text { for } i j k \neq i^{\prime} i^{\prime} k^{\prime} \text {. alternately } l \neq m
\end{gathered}
$$

This results in the formation of a diagonal (a) mass matrix. Similar results follow from the 
same ideas to form diagonal (b) and (c) mass matrices.

All that remains at this point is the formation of the (d) mass matrix. In the case of an orthogonal mesh orthogonality of the vectors in the basis functions will cause the (d) all of the (d) mass integrals to evaluate to 0 . This will make the (d) mass matrix empty and consequently make the mass matrix diagonal. On non-orthogonal meshes the basis vectors are no longer orthogonal, and no longer evaluate to 0 . Also, since the (d) mass integrals involve basis functions on different sets of interpolation points, the trick of using interpolation points for integration points no longer works. However, the basis functions still consist mostly of Legendre polynomials on the Gauss-Lobatto interpolation points. This makes using Gauss-Lobatto integration in all three dimensions a reasonable choice if one wishes to increase sparsity. While this choice does not make the $(\mathrm{d})$ mass matrix diagonal, it can be shown that the integration rule evaluates to 0 for some combinations of basis functions that are pointing in different directions. This integration rule also has the advantage of keeping the order of integration consistent with the other three integration rules.

\section{Experimental Validation}

While the sparsity results of the directional mass lumping approximation work well for reducing the total work in simulating Maxwell's wave equation, they leave the accuracy of the approximations in question. It is with this in mind that we completed a numerical experiment in order to provide some validation for this approximation.

The experiment consisted of simulating TEM waves traveling down a coaxial cable using the standard method with an exact integration rule and a directional mass lumping integration rule. In both cases $2^{\text {nd }}$ order basis functions were used as well as $2^{\text {nd }}$ order geometry in order to better approximate the cylindrical walls of the cable. PEC boundary conditions were applied to the inner and outer walls of the cable while an ABC was applied at one of the ends in order to terminate the wave. Finally, a time dependent voltage source was applied on the other end of the cable in order to drive the TEM wave.

The ideal cable was roughly 14 waves long with an inner radius of roughly 1 wave and an outer radius of roughly 2 waves in normalized space. The hexahedral mesh approximated this geometry by using 50 elements along the length of the cable, 5 elements to cross from the inner radius to the outer radius, and 14 elements to go around each of the radii. While this may seem a bit coarse, the $2^{\text {nd }}$ order basis functions and element geometries make it reasonable.

These simulations were carried out in time steps of $0.1 \mathrm{u}$ ( $\mathrm{u}$ a unit of time normalized to the speed of light) and were run for a total of 1000 time steps. At each time step the mass matrix was 'inverted' using the PCG method with a simple diagonal preconditioner and a tolerance of 1e-10. At 50 time step increments the the electric field values were written out to disk. The magnitudes of these values were compared to magnitudes in the exact solution of the coaxial cable problem to compute error. Finally, the maximum of the error in each time step was computed and plotted.

In order to carry out these simulations a software package from LLNL called EMSolve v2 was modified to introduce directional mass lumping approximations. This software had all of the other capabilities (higher order bases, higher order geometry, time dependent sources, etc.) necessary to carry out these simulations.

\section{Results}

The first result is simply a best case example of how much computation time can be saved by using a directional mass lumping approximation. Using a small orthogonal mesh and $2^{\text {nd }}$ order elements it would be reasonable to expect roughly 100,000 Degrees Of Freedom (DOF). Using the standard method without mass lumping one can expect roughly 20 nonzero elements in the mass matrix per DOF, and to invert this matrix one would expect to need roughly 20 PCG iterations. This comes out to a cost of roughly 40 million multiplications per time step. However, in the case where directional mass lumping is used, the mass matrix is diagonal so it only has 1 non-zero element per DOF, and only 
takes roughly 1 PCG iteration of work to invert. This translates to 100,000 multiplications per time step, which is $1 / 400^{\text {th }}$ the amount of work, which eliminates the mass matrix inversion as the dominating computation cost of the simulation.

A more realistic example of the gain in computational efficiency is calculated using the coaxial cable problem. In this case the the mesh is no longer orthogonal and the mass matrix is no longer diagonal. However, as mentioned before, the added sparsity still allows for gains in efficiency. The sparsity in the mass matrices is measured by counting the number of non-zero entries. This number is directly related to the amount of work that must be done for each PCG iteration. In the case without mass lumping the mass matrix had a total of 5,201,424 non-zero entries, while the mass lumped case only had 860,016. This alone translates roughly to $1 / 6^{\text {th }}$ of the work involved the mass matrix 'inversion'. Additionally, on average, the non-mass lumped matrix took 21 PCG iterations to converge, whereas the mass lumped matrix took only 9 iterations. The combination of these two effects leaves the mass matrix inversion with roughly $1 / 12^{\text {th }}$ the amount of work.

The other result is a measure of the error added by using directional mass lumping in the coaxial cable problem. The following plot shows the max error as a function of time with and without mass lumping.

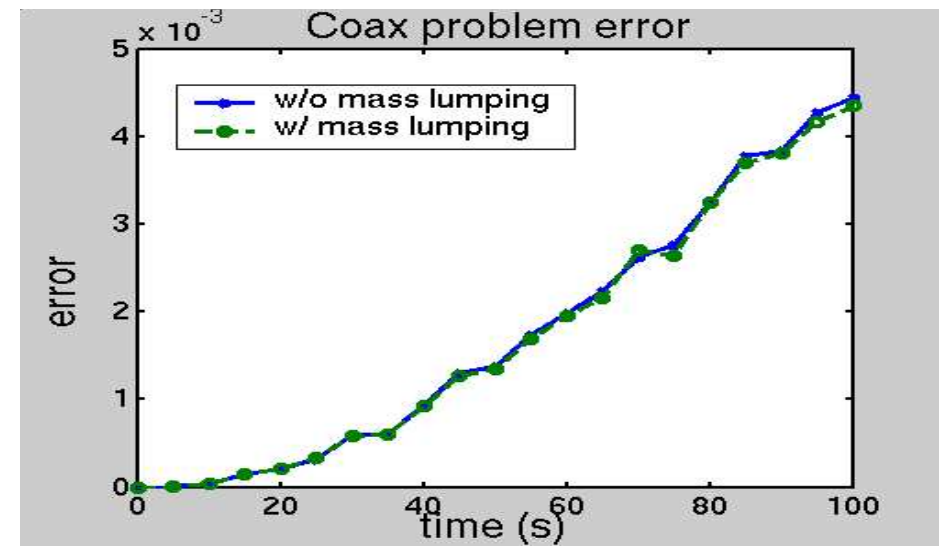

It is readily apparent that the directional mass lumping method did not add any substantial error in this particular simulation.

\section{Conclusions}

The directional mass lumping approximation was outlined and applied to the Nedelec bases for Maxwell's wave equation. In the case of orthogonal meshes the approximation was found to eliminate the mass matrix inversion as a computational bottleneck. The approximation was also tested on the coaxial cable problem and was found to improve the computational cost for the mass matrix inversion by an order of magnitude, while having a negligible effect on the error of the simulation. This preliminary result was by no means a complete validation of the directional mass lumping method, however it does illustrate the potential for this method to have a negligible impact on the error.

\section{References}

[1] G. Rodrigue and D. White. A vector finite element time-domain method for solving Maxwell's equations on unstructured hexahedral grids. SIAM J. Sci. Comp., V23(N3):683706, Oct. 2001.

[2] J. C. Nedelec. Mixed finite elements in R3. Numer. Math., 35:315-341, 1980.

[3] G. Cohen and P. Monk. Gauss Point Mass Lumping Schemes for Maxwell's equations. Numer. Meth. PDE., 14:63-88, 1998. 